\title{
Posterior Reversible Encephalopathy Syndrome in COVID-19 Disease: a Case-Report
}

\author{
Laura Llansó $^{1}$ (D) Xabi Urra ${ }^{1,2}$ (D) \\ Accepted: 20 August 2020 / Published online: 26 August 2020 \\ (C) Springer Nature Switzerland AG 2020
}

\begin{abstract}
Posterior reversible encephalopathy syndrome (PRES) is a clinical syndrome that can include headache, altered consciousness, visual disturbances, and seizures, usually related to autoregulatory cerebral failure and hypertension. The neuroimaging is essential to diagnosis, showing white matter vasogenic edema in posterior areas. We present a case of a 66-year-old woman with severe pneumonia by SARS-CoV-2 who developed a posterior reversible encephalopathy syndrome with a typical clinical and radiological presentation, after being treated with anti-interleukin treatment (anakinra and tocilizumab) following local guidelines. We report a case of posterior reversible encephalopathy syndrome in a patient with COVID-19 disease, possibly related to anti-IL-1 or anti-IL-6, suggesting that anti-interleukin treatments may cause this syndrome, at least in patients with predisposing conditions such as infections and hydroelectrolytic disorders.
\end{abstract}

Keywords Posterior reversible encephalopathy syndrome (PRES) $\cdot$ COVID-19 $\cdot$ Anakinra $\cdot$ Tocilizumab $\cdot$ Immunomodulators

\section{Introduction}

A 66-year-old woman with COVID-19 presented with adult respiratory distress syndrome (ARDS). Besides bilateral pneumonia, she developed multiple complications such as cardiorespiratory arrest, bacterial superinfection, hyponatremia, massive hemoptysis requiring embolization, and acute renal injury. She was started on lopinavir/ritonavir, hydroxychloroquine, and azithromycin. After radiological pulmonary progression, anti-IL-1 (daily anakinra) and anti-IL-6 (single dose of tocilizumab) were started, following local and hospital guidelines. These drugs are recommended in COVID-19 when there is clinical, blood test, or radiological progression, to avoid an excessive immunological systemic

This article is part of the Topical Collection on COVID-19

Laura Llansó

LLANSO@clinic.cat

Xabi Urra

XURRA@clinic.cat

1 Department of Neurology, Hospital Clínic, C/ Villarroel 170, 08036 Barcelona, Catalonia, Spain

2 Comprehensive Stroke Center, Department of Neuroscience, Hospital Clínic, August Pi i Sunyer Biomedical Research Institute (IDIBAPS), Barcelona, Catalonia, Spain response to the virus, which is thought to worsen pulmonary infiltrates and disease prognosis. Ten days after the initiation of immunodepressants, she developed altered mental status without fever, previous headache, or visual disturbances.

\section{Case Presentation}

At the examination, the patient opened eyes to painful stimuli, had no verbal response, and showed withdrawal response to pain (Glasgow Coma Scale 7). The blood tests showed stable hyponatremia $(130 \mathrm{mEq} / \mathrm{L})$ and leukocytosis without any other significant findings. Her vitals were within normal limits, and blood pressure had been mildly increased during the previous $12 \mathrm{~h}$ with a maximum systolic pressure of $160 \mathrm{mmHg}$. Electrocardiogram showed sinus rhythm and had not atrioventricular node blocks. A CT scan with angiography was performed. There was no large vessel occlusion, no perfusion alterations, and the baseline CT (Fig. 1) showed temporooccipital white matter hypodensity with symmetric obliteration of the sulci in that region.

Considering the infectious background, the immunomodulatory treatments, modest hypertension in the hours before the symptoms, and the distribution of the lesions on the CT scan, the most likely diagnosis is posterior reversible encephalopathy syndrome (PRES) [1-5]. Hypertension plays a vital role in 
Fig. 1 CT baseline scan showed temporo-occipital symmetric white matter hypodensity

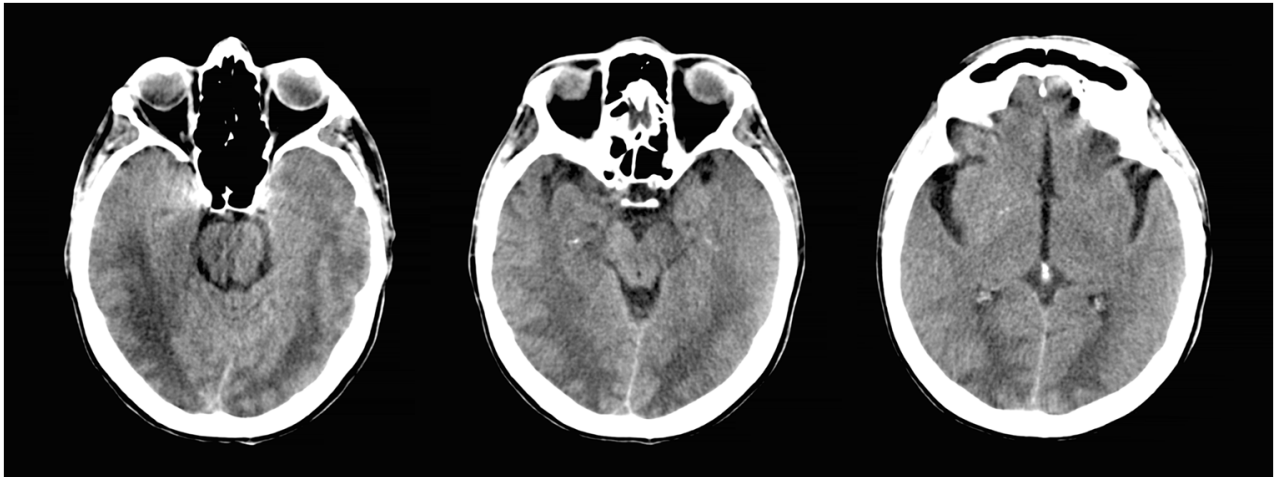

the disease due to a failure in cerebral blood flow autoregulation, and in this case, rapid rise or fluctuations in blood pressure from baseline may have been harmful, despite not being severely high [2]. The electroencephalogram showed focal slowing and epileptiform discharges in both occipital areas and ruled out nonconvulsive status epilepticus. The symptoms improved over the following days after tight control of blood pressure with labetalol infusion and discontinuing anakinra.

After 1 week, radiological infiltrates worsened and a blood test showed increased acute phase reactants. In this context, a diffuse alveolar hemorrhage was diagnosed by bronchoalveolar lavage, with suspicion of hemophagocytic syndrome. She required red blood cell transfusions and immunosupressants were restarted, as well as mechanical ventilation. The CT pulmonary scan showed worsening of infiltrates and presence of an intrapulmonary hematoma. Finally, the patient had a torpid evolution to multiorgan failure and death.

\section{Conclusions}

The absence of fever and radiological findings did not suggest an encephalitic cause of the symptoms. The serum sodium levels were only slightly decreased and had been stable for the previous days without intravenous infusion of sodium. Thus, hyponatremia was not assumed to be the cause of the sudden loss of consciousness. The cardiorespiratory arrest happened a month before the event while intubated, and it was secondary to a mucus plug after a period of desaturation and bronchospasm. It lasted less than $5 \mathrm{~min}$ and ended as the mucus plug was removed by fibrobronchoscopy. The postanoxic cerebral damage was prevented by treating fever; avoiding systemic hypotension, hypoxemia, or glycemic disbalance; and continuing renal replacement therapy with hemodiafiltration instead of hemodialysis to prevent large changes in volemia. One week later, sedation was stopped and a tracheostomy was placed, and the patient progressively awakened up to a normal state of consciousness without focal neurologic signs. The timeline and posterior complete recovery from the respiratory arrest cannot explain the current episode as hypoxic-ischemic encephalopathy.

PRES has been associated with immunosuppressive and cytotoxic therapies such as platinum-containing drugs, (R)CHOP regimens, gemcitabine, cyclosporine, tacrolimus, sirolimus, and interferon therapies. Also, agents that target angiogenesis such as bevacizumab (anti-VEGF) and tyrosine kinase inhibitors (TKI) against VEGF receptor (pazopanib, sorafenib, sunitinib) have been described as risk factors $[3$, 4]. Prior exposure to the predisposing drug does not appear to be protective, and patients can develop PRES even after several months after exposure [2]. Furthermore, the disorder has been associated with both acute and chronic renal disease, as was the case in our patient, and medical conditions such as hyponatremia or pulmonary infection could exacerbate the neurological findings.

Despite not being described yet, the occurrence of PRES a few days after anti-interleukin (IL-6 or IL-1) treatments which were given in this patient, raises the possibility that these kinds of immunomodulatory agents may also favor PRES.

\section{Compliance with Ethical Standards}

Conflict of Interest The authors declare that they have no conflict of interest.

Ethical Approval and Informed Consent Consent for publication was obtained from the next of kin (daughter). Approval from the Hospital's IRB was provided for this study.

\section{References}

1. Fugate JE, Claassen DO, Cloft HJ, Kallmes DF, Kozak OS, Rabinstein AA. Posterior reversible encephalopathy syndrome: associated clinical and radiologic findings. Mayo Clin Proc. 2010;85: 427-32.

2. Gao B, Lyu C, Lerner A, McKinney AM. Controversy of posterior reversible encephalopathy syndrome: what have we learnt in the last 20 years? J Neurol Neurosurg Psychiatry. 2018;89:14-20. 
3. Allen JA, Adlakha A, Bergethon PR. Reversible posterior leukoencephalopathy syndrome after bevacizumab/FOLFIRI regimen for metastatic colon cancer. Arch Neurol. 2006;63: 1475-8.

4. Ito $\mathrm{Y}$, Arahata $\mathrm{Y}$, Goto $\mathrm{Y}$, et al. Cisplatin neurotoxicity presenting as reversible posterior leukoencephalopathy syndrome. AJNR Am J Neuroradiol. 1998;19:415.
5. Schwartz RB, Jones KM, Kalina P, Bajakian RL, Mantello MT, Garada B, et al. Hypertensive encephalopathy: findings on CT, MR imaging, and SPECT imaging in 14 cases. AJR Am J Roentgenol. 1992;159:379-83.

Publisher's Note Springer Nature remains neutral with regard to jurisdictional claims in published maps and institutional affiliations. 